\title{
LA CONCILIATION, LA MAL-AIMÉE DES JUGES
}

\author{
par Jacques Poumarède, \\ professeur émérite à l'Université Toulouse I Capitole, \\ conciliateur de justice
}

Dans notre société postmoderne, la tendance est aux MARL (modes alternatifs de règlement des litiges). Face à une justice enfermée dans ses temples, ligotée par ses rituels et ses procédures, rendue opaque sinon aveugle par la technicité du droit et submergée par la marée contentieuse, la solution n'est-elle pas d'encourager les transactions, les arrangements entre les parties elles-mêmes, sans coûts ni lenteurs, sans formalisme et sous le regard bienveillant mais éclairé d'un tiers chargé de les réunir, c'est-à-dire de les concilier, selon l'étymologie latine (conciliare) ?

En France, à l'époque contemporaine et pour ce qui concerne l'ordre judiciaire, le mouvement des MARL a débuté modestement avec l'institution par le décret du 20 mars 1978 des «conciliateurs » chargés de résoudre bénévolement et à l'amiable de petits litiges civils relevant du tribunal d'instance mais avant toute introduction d'instance. La loi de 1995 relative à l'organisation des juridictions et de la procédure a intégré ces conciliateurs dans le processus judiciaire et un décret de 1996 a autorisé les juges d'instance à leur déléguer le droit de concilier les parties dans des instances déjà engagées. Ces mêmes textes ont aussi permis la nomination de «médiateurs » dans des domaines spécialisés telles les relations familiales ou conjugales.

Aujourd'hui la conciliation comme la médiation paraissent des procédures bien installées et en expansion, d'autant plus que l'Europe du droit pousse à la roue. Une directive européenne encourage depuis 2008 le recours aux MARL; elle vient d'obtenir sa transposition en droit français par l'ordonnance du 16 novembre 2011. La doctrine s'est emparée de la question et a produit une abondante littérature, ce qui ne va pas sans 


\section{Jacques Poumarède}

entretenir quelques obscurités et pas mal d'ambiguïtés ${ }^{1}$. La différence entre la conciliation qui se préoccupe de réunir les parties au litige et le rôle du médiateur qui, si l'on en juge par l'étymologie (du latin : mediare) devrait se tenir au milieu d'elles n'est pas des plus claires.

On peut se demander aussi comment ce déferlement des MARL est vécu par l'institution judiciaire elle-même. L'esprit de réserve qui anime le corps judiciaire laisse peu transparaitre les opinions, mais les chiffres parlent. D'après le Compte général de la justice, en 2008, 1794 conciliateurs de justice ont traité 112828 conflits et en ont concilié 67245 soit un taux de 59,6\%, en progression de $+1 \%$ sur 2007: un résultat, somme toute, satisfaisant. Toutefois, un chiffre moins connu tempère ce constat: sur les 63616 conciliations enregistrées, 6843 seulement $(10,75 \%)$ ont été conclues sur délégation du juge d'instance ou de proximité. Force est de constater que cette activité modeste et bénévole n'a pas la faveur des juges. Le conciliateur qui s'exprime ici a eu l'occasion de constater que devant les tribunaux d'instance de la cour d'appel de Toulouse, les juges professionnels ne délèguent pratiquement jamais en conciliation et les juges de proximité guère davantage. La conciliation est restée pour l'essentiel un acte extrajudiciaire.

La méfiance des juges à l'égard des modes alternatifs de règlement des litiges que l'on présente volontiers comme un emprunt récent à la pratique anglo-saxonne de l'alternative dispute resolution $(A D R)^{2}$ est en réalité une attitude bien française qui accompagne depuis deux siècles certains aspects des "Désunions de la magistrature", thème du colloque. Pour en rendre compte, nous proposons de suivre l'histoire de la conciliation en voyant d'abord, après un bref engouement sous la Révolution, la désaffection dont elle a fait l'objet au cours du XIXe siècle et au XXe jusqu'à la veille de la II ${ }^{e}$ Guerre mondiale, puis nous évoquerons les conditions de sa modeste réapparition dans le dernier quart du XXe siècle.

\footnotetext{
${ }^{1}$ Symptome de ces incertitudes, les hésitations sur la dénomination du phénomène : aux tenants des MARL s'opposent les adeptes des «modes alternatifs de résolution des conflits» (MARC); dans une bibliographie foisonnante, on distinguera P. CHEVALIER, Y. DESDEVISES, Ph. MILBURN (sous la dir.), Les modes alternatifs de règlement des litiges: les voies nouvelles d'une autre justice, Paris, La Documentation française, 2003, $288 \mathrm{p}$.

${ }^{2}$ E. LE ROY (dir.), La conciliation et les modes para-judiciaires de règlement des litiges. Expériences françaises et nord-américaines, Paris, Laboratoire d'anthropologie juridique de Paris, Bordeaux, Association d'études et de recherches de l'École nationale de la magistrature, coll. "Essais et recherches judiciaires », 1989.
} 


\section{I - les avatars de la conciliation (1790-1939)}

L'antinomie entre l'exercice de la justice par des juges professionnels et la pratique de la conciliation est apparue très clairement au cours des débats que la Constituante a tenus sur la réforme de l'organisation judiciaire et qui ont abouti à la loi des 16-24 août 1790. En réaction contre les modes de fonctionnement des institutions judiciaires de l'Ancien Régime, la conciliation a été présentée comme la «pierre de touche» qui allait permettre de "déjudiciariser» de nombreux conflits et, sous l'influence prépondérante de Jacques-Guillaume Thouret, l'Assemblée a adopté en matière civile un ensemble de principes destinés à restituer la justice d'État aux citoyens et à résoudre les conflits par les seules vertus de la raison. Dès $l^{\prime}$ article $1^{\text {er }}$ de la loi, il est fait appel en priorité à l'arbitrage comme « étant le moyen le plus raisonnable de terminer les contestations entre les citoyens ", et dans le titre $X$ ce principe est mis en œuvre par l'instauration «des bureaux de paix et du tribunal de famille».

L'idée de confier le règlement des conflits familiaux à des assemblées de parents, d'amis et de voisins n'était pas neuve, elle était pratiquée de tout temps dans de nombreux pays de coutume comme de droit écrit, mais les Constituants l'ont imposée partout en lui donnant un cadre uniforme. Avec les bureaux de paix, la « philosophie conciliatrice » qui anime la révolution judiciaire de 1790-1791 dépasse largement la sphère domestique. Sans doute, les juges de paix institués par le titre III de la loi avaient des prédécesseurs parmi les «faiseurs de paix» (vredemakers) hollandais ou, plus vraisemblablement, en la personne du juge auditeur du Châtelet de Paris affecté au règlement des petits litiges. Ils ont reçu, eux aussi, une compétence contentieuse sur «toutes les causes purement personnelles et mobilières, sans appel jusqu'à la valeur de cinquante livres, et à charge d'appel jusqu'à la valeur de cent livres » (art. 9), ainsi qu'une juridiction gracieuse sur tout un ensemble de questions juridiques de la vie ordinaire. Mais ces nouveaux juges élus par l'assemblée des citoyens actifs du canton tirent leur principale originalité de la fonction de conciliation qui leur a été attribuée par le titre $X$ de la loi (articles 1 à 11). Dans toutes les causes civiles excédant sa compétence, le juge de paix, assisté de deux à quatre assesseurs, devait entendre les dires des parties et sans se prononcer sur le fond tenter de leur faire trouver un accommodement acceptable consigné dans un accord écrit. Le tribunal de district ne pouvait être saisi et une procédure n'était engagée que si ce préliminaire de conciliation avait échoué. La mission assignée à ce bureau de paix était clairement de faire prévaloir le 


\section{Jacques Poumarède}

bon sens et l'harmonie sociale entre citoyens et de leur épargner les désagréments d'un procès ou, pire, les excès de la "fureur thémistique »³.

L'importance de cette obligation d'une conciliation préalable a paru si grande aux hommes de la Révolution qu'ils en firent une règle de la constitution de 1791 (ch. V, art.6), rappelée dans l'article 215 de la constitution de l'an III, et réitérée en des termes très explicites dans celle de l'an VIII : «Art. 60 - Chaque arrondissement communal a un ou plusieurs juges de paix, élus immédiatement par les citoyens pour trois années. Leur principale fonction consiste à concilier les parties, qu'ils invitent, dans le cas de non-conciliation, à se faire juger par des arbitres ».

Il n'est pas le lieu de rendre compte ici de la manière dont les juges et les bureaux de paix se sont acquittés de cette mission au cours de l'époque révolutionnaire ; évoquons plutôt son évolution à partir de la codification ${ }^{4}$.

Le préliminaire de conciliation a été maintenu dans le code de procédure civile de 1806 en ces termes: "Art. 48: Aucune demande introductive d'instance ne sera reçue dans les tribunaux de première instance que le défendeur ou les parties d'un commun accord n'aient été préalablement appelées en conciliation devant le juge de paix». Mais la formulation péremptoire de cette disposition ne doit pas faire illusion, car le contexte était complètement transformé depuis les réformes de l'an VIII et spécialement les lois du 27 ventôse sur la nouvelle organisation judiciaire. Une pyramide de tribunaux et de cours avait été dressée et peuplée par un corps de magistrats nommés par le pouvoir exécutif et réinvestis de la fonction régalienne de juger; dans le même temps, le monde de la robe s'était recomposé avec le rétablissement des avocats et des avoués. L'heure

3 J. LEONNET, «Une création de l'Assemblée constituante: la conciliation judiciaire », Une autre justice, 1789-1799, R. BADINTER (dir.), chap. XIII, Paris, Fayard, 1989 ; J.-J. CLERE, « Recherche sur l'histoire de la conciliation en France aux XVIII ${ }^{\mathrm{e}}$ et $\mathrm{XIX}^{\mathrm{e}}$ siècles », Mémoires de la Société pour l'histoire du droit des anciens pays bourguignons, comtois, romands, 1989, fasc. 46, p. 189 ; sur le débat révolutionnaire autour des procédures conciliatoires on lira aussi les développements de Jean-Pierre ROYER dans Histoire de la justice du XVIII à nos jours, Paris, PUF, 2010, 4 ème édition, p. 260 à 270 ; ainsi que Jacques KRYNEN, L'état de justice France, XIII ${ }^{e}-X X^{e}$ siècle, II, L'emprise contemporaine des juges, Paris, NRF - Ed. Gallimard, 2011, p. 23 à 29.

${ }^{4}$ Sur la justice de paix, on renvoie à l'ouvrage classique de Guillaume METAIRIE, Le monde des juges de paix de Paris (1790-1838), Paris, Éd. Loysel, 1994, complété par les réflexions du même auteur dans La justice de proximité, une approche historique, Paris, PUF, Léviathan, 2004, p. 108 à 121; voir également les contributions réunies dans J.-G. PETIT (dir.), Une justice de proximité : la justice de paix 1790-1958, Paris, PUF, coll. Droit et justice, 2003. 
n'était plus aux «niaiseries métaphysiques » selon la formule attribuée par Fouché à Bonaparte.

Au cours des débats sur le code de procédure, bien que le rapporteur, Jean-Baptiste Treilhard, se soit montré favorable, la conciliation est apparue à bon nombre d'orateurs comme une «vaine formalité » et, au Tribunat comme au Corps législatif, il y eut des voix pour sa suppression ${ }^{5}$. Toutefois, le principe était inscrit dans la constitution et on avait maintenu les juges de paix en réduisant leur champ d'intervention. Le code leur a donc laissé le soin de conduire la tentative de conciliation mais d'une manière très encadrée par les articles 49 et suivants. Elle a pris une allure procédurale avec l'obligation pour le demandeur de faire délivrer au défendeur une citation par un huissier (art.50) dans un délai de trois jours au moins (art. 51); les parties devaient comparaître en personne, mais en cas d'empêchement pouvaient se faire représenter par un fondé de pouvoir (art. 52). Le juge de paix devait tenter de les concilier et, s'il n'y parvenait pas, délivrait un certificat de non-conciliation qui leur permettait de poursuivre l'action devant le tribunal civil compétent.

Surtout, un certain nombre de demandes ont été exemptées par l'article 49 : toutes celles qui intéressaient les personnes publiques comme l'État, le Domaine, les communes ou les personnes privées incapables de transiger comme les mineurs non-émancipés, les interdits, les curateurs et les femmes mariées. La même dispense a bénéficié aussi aux demandes en matière de commerce, aux litiges concernant plus de deux défendeurs et à ceux « qui requièrent célérité » (art. 49-2).

La jurisprudence s'est employée à élargir ces failles. Les cours d'appel et la Cour de cassation ont d'abord refusé de reconnaître le caractère d'ordre public au préliminaire de conciliation, empêchant ainsi que son défaut, même soulevé d'office, puisse entraîner la nullité de l'action ${ }^{6}$. Le défaut de comparution devant le juge de paix n'était sanctionné que par une simple amende longtemps fixée à la modeste somme de dix francs. Mais c'est surtout l'accueil réservé par les juges de première instance à l'exemption

\footnotetext{
${ }^{5}$ Voir la position de Treilhard et les opinions contraires dans J.-G. LOCRE, La législation civile, commerciale et criminelle de la France, Paris, 1830, t. XXI, p. 247-249; J. L. HALPERIN, «Le code de procédure de 1806: un code de praticiens ? », 1806 - 1976 - 2006, De la commémoration d'un code à l'autre : 200 ans de procédure civile en France, (L. CADIET et G. CANIVET, dir.), Paris, Litec, 1989, p. 28.

${ }^{6}$ Un débat a eu lieu sous l'Empire et sous la Restauration, mais il a été tranché par un arrêt du 16 fév. 1826 de la Cour de cassation (Req., Lugo, D. P., 26.1.1974), rappelé à différentes reprises, dont Req. 6 déc. 1892, D. P., 93.1. 479.
} 


\section{Jacques Poumarède}

pour célérité qui a vidé cette procédure gracieuse de l'essentiel de sa substance. Les tribunaux d'arrondissement ont pris l'habitude d'accorder très libéralement cette exemption sur la base d'un permis d'assigner à bref délai délivré sans motivation particulière par le président. Après quelques hésitations, la Cour de cassation a couvert cette pratique.

Le résultat est une désaffection continue pour cette procédure gracieuse comme l'attestent quelques chiffres glanés dans les comptes généraux de la justice civile publiés depuis la monarchie de Juillet. En 1840, 82419 affaires ont été portées en conciliation devant le juge de paix et ont donné lieu à 41517 conciliations, soit un taux de 50,37\%. En 1856, 55115 préliminaires pour 18584 conciliations effectivement réalisées (33,71\%); en 1890, 30153 pour 6679 conciliations (22,15\%) et en 1906, 13519 pour $3793(28,05 \%)$. Alors que le volume général du contentieux porté devant les tribunaux de première instance n'a cessé de croître, le nombre des saisines de l'article 48, pourtant obligatoires, s'est effondré et, qui plus est, le pourcentage de réussite est tombé de moitié.

Il est évident que cette désaffection s'est nourrie de l'attitude négative des hommes de loi. Les auxiliaires de justice, avocats et avoués, détestaient la conciliation qu'ils voyaient comme un détournement d'éventuels plaideurs et les magistrats eux-mêmes ne s'y intéressaient pas. La reconstitution du corps judiciaire a laissé de côté les juges de paix pour des raisons aussi bien sociales que statuaires. Ces petits juges, « hommes à tout faire» des cantons étaient bien éloignés des notables qui peuplaient les cours et tribunaux. Pour ces derniers, l'office du magistrat était d'exercer l'art de juger : une fonction régalienne, un imperium. La recherche laborieuse d'une transaction n'était pas à la hauteur de leur dignité. Les préliminaires de conciliation que leur imposait le code n'étaient perçus que comme des pertes de temps et le fait que la conciliation ait été une invention révolutionnaire n'a pas amélioré leur opinion. Les rapports des chefs de cour sur l'activité et les capacités des juges de paix sont souvent très condescendants.

La doctrine a fait chorus. Dans la première édition du Dictionnaire général de... jurisprudence, des frères Dalloz (1835), l'article "Conciliation" s'ouvre par une phrase qui exprime clairement cet état d'esprit : «C'est à l'assemblée constituante que nous devons l'introduction en France du préliminaire de conciliation, institution empruntée aux Anglais, et qui, exercée par des magistrats habiles, peut prévenir une foule de procès et d'inimitiés, mais ce n'est le plus souvent qu'une vaine et onéreuse formalité ». Dès 1828, un des premiers commentateurs du code de 1806, 
Pierre Boncenne, professeur à la faculté de droit de Poitiers, tout en reconnaissant que « la conciliation fut prescrite pour le maintien de la paix entre citoyen", constate que "la jurisprudence ne la favorise guère. [...] Cette indifférence a achevé de décolorer la conciliation; elle n'est plus, à vrai dire, qu'une de ces idées qui, n'étant ni entièrement rejetées, ni entièrement adoptées, restent dans la circulation, sans tirer à conséquence ${ }^{7}$. Dix ans plus tard, dans son Traité de la compétence des juges de paix, l'avocat Jacques Curasson reprend le même point de vue, mais en mettant cette évolution sur le compte d'une opposition entre les villes et les campagnes : «Je suis loin de contester l'utilité de ce préliminaire: les idées d'indépendance, qui dominent toutes les classes de la société, sont, il est vrai, peu compatibles avec les mesures conciliatrices, et l'esprit de chicane, surtout dans les villes, a fait dégénérer la tentative du bureau de paix en une pure formalité ; cependant il est des campagnes où la parole d'un homme investi d'une juste considération, peut produire les plus heureux effet ${ }^{8}$. Mais, ajoute-il, pour concilier d'une manière équitable, encore faut-il être à même d'apprécier les droits des parties et «si j'en juge d'après l'expérience, la conciliation exige autant et même plus de lumières que le jugement de la plupart des contestations soumises aux juges de paix». Et Curasson de s'interroger : dépourvus de diplôme, ces derniers sont-ils capables d'exercer ce ministère ?

Le discrédit jeté sur le préliminaire de conciliation a eu pourtant un effet paradoxal sur l'activité des juges de paix. Aussi curieux que cela puisse paraître, le code de procédure civile de 1806 n'avait pas prévu de conciliation préalable pour les affaires relevant de leur propre compétence d'attribution. Les juges ne pouvaient être saisis que par une citation délivrée par huissier et ne devaient se prononcer qu'en plein contentieux. Cette situation déplaisait à bon nombre de juges de paix, frustrés de voir leur activité conciliatrice se réduire, alors qu'ils se dévouaient à l'instar du bon juge Clousier qui, dans Le Curé de village, était estimé des paysans «à cause du désintéressement paternel avec lequel il accordait leurs différends et leur donnait ses conseils dans leurs moindres affaires ${ }^{9}$. Aussi, certains d'entre eux ont pris l'habitude d'interdire aux huissiers de leur canton de délivrer

\footnotetext{
${ }^{7}$ P. BONCENNE, professeur à la faculté de droit de Poitiers, Théorie de la procédure civile, Poitiers, Librairie Catinea, 1828, t. I, p. 297.

8 J. CURASSON, Traité de la compétence des juges de paix, Dijon, V. Lagier, 1839 , t. I, introduction, p. XII.

${ }^{9}$ H. DE BALZAC, CEuvres complètes, Paris, Gallimard, coll. La Pléïade, t. IX, p. 812.
} 


\section{Jacques Poumarède}

aucune citation, avant qu'ils n'aient eux-mêmes convoqué les parties officieusement par une simple lettre. Cette pratique connut rapidement d'excellents résultats et elle fut intégrée dans l'article 17 de la loi du 25 mai 1838 portant réforme de la justice de paix, mais à titre facultatif ${ }^{10}$; puis, sous le Second Empire, une loi du 2 mai 1855 l'a rendue obligatoire.

À la différence du préliminaire de conciliation, la « petite conciliation » a suscité un extraordinaire engouement qui s'est chiffré par millions. Dès 1856, les greffiers des justices de paix ont émis 3360000 convocations, dénommées «billets d'avertissement», qui donnèrent lieu à 1450000 conciliations ( $43 \%)$, et à presque autant d'abandons du litige par les parties $(38 \%)$. Les juges de paix n'eurent à juger réellement que 423000 affaires (12\%). L'optimum fut atteint en 1865 avec 3556000 avertissements. Mais dès le début de la III ${ }^{e}$ République une décrue s'amorce avec la loi du 23 août 1871 qui prescrit de rédiger les billets sur papier timbré. Le nombre tombe au-dessous des 2000000 en 1879, il se situe autour de 1500000 au tournant de 1900. En 1913, il n'est plus que de 1142000 et en 1938, de $534569^{11}$. Les taux de réussite de la «petite conciliation » ont baissé également mais plus modérément : ils se situent autour de $38 \%$ au début du XIXe siècle, pour se stabiliser à $30 \%$ du nombre total des avertissements dans l'entre-deuxguerres $^{12}$.

Le déclin de la conciliation, la grande comme la petite, n'est pas seulement dû à l'hostilité des hommes de loi et au manque d'appétence des justiciables, les juges de paix ont eu leur part de responsabilité. Les commentateurs n'ont pas manqué de faire observer que l'accroissement constant des tâches mises à la charge de ces petits juges à la fin du siècle

10 J.-B. DUVERGIER, Collection complète des lois, décrets, ordonnances, règlements..., Année 1838, Paris, Bousquet, 1839, p. 359 ; la circulaire du garde des Sceaux du 6 juin, précise : «Je ne vois que de l'avantage à ce que cet usage soit maintenu là où il existe, et à ce qu'il soit introduit dans les cantons où il n'a pas été encore établi. C'est afin de laisser à cet égard aux juges de paix tout le mérite de l'initiative, et de leur permettre d'apprécier les circonstances dans lesquelles la remise de cet avis serait utile ou superflu ».

${ }^{11}$ H. VIEILLEVILLE, Le rôle judiciaire et social du juge de paix, thèse droit, Paris, 1944 , p. 82-83.

${ }^{12}$ Ces pourcentages globaux recouvrent en fait des disparités assez nettes entre ressorts judiciaires, comme le montrent les travaux de cartographie statistique (maping) de Gilles ROUET ; voir sa contribution : «La justice de paix en France entre 1834 et 1950 : une exploration spatiale », dans J.-G. PETIT (dir.), Une justice de proximité... ouv. cité, p. 67-99. L'analyse des cartes réalisées fait apparaître une nette dichotomie Nord/Sud qui montre les « anciens pays de droit écrit » moins bien disposés aux accommodements que les « ci-devant pays de coutumes ». 
(saisies-arrêts sur salaire, constat et réparation des accidents du travail, augmentation des actes de juridiction gracieuse) leur a laissé de moins en moins de temps à consacrer à une activité négligée par les magistrats supérieurs $^{13}$. Dans le même temps, leur statut commençait à évoluer. Alors qu'aucun diplôme n'était exigé, les recrutements des juges de paix sont devenus plus sélectifs à la fin du XIX`e siècle avec l'exigence d'une expérience professionnelle préalable, puis, à l'instigation de Louis Barthou une loi du 1918 a institué un recrutement sur examen d'aptitude et la réforme judiciaire de 1926 a exigé des candidats une licence en droit et un stage pratique dans un greffe ou une étude de notaire. Les juges de paix vont se mettre à ressembler de plus en plus aux magistrats inamovibles des cours et tribunaux, mais avec une échelle des rémunérations moins haute. Dans ces conditions, la justice paternelle et immédiatement accessible, voulue par les révolutionnaires, était-elle encore compatible avec la «fin des terroirs ${ }^{14}$, avec une société en voie d'urbanisation et soumise à une technicité de plus en plus poussée des rapports juridiques?

\section{II - Mort et résurrection (laborieuse) de la conciliation}

À la Libération, s'est constituée, sous une forme associative, l'Union fédérale des magistrats avec pour objectif la défense des intérêts professionnels de la magistrature et le souci de rassurer voire de ressouder un corps assez bousculé au cours des « années noires » du régime de Vichy. Dès sa fondation en décembre 1945, L'UFM a admis en son sein les juges de paix et son bulletin, Le Pouvoir judiciaire, leur a consacré quelques tribunes. Dans le $n^{\circ} 4$ (1946), deux d'entre eux: Maxime Pépin et Guy Fau, membres du bureau exécutif de l'UFM se livrent sous le titre "Avenir des justices de paix » à un plaidoyer en faveur du maintien des juridictions cantonales et de leurs titulaires mais dans des attributions assimilées aussi bien au niveau des aptitudes que du statut et des rémunérations à celles des autres magistrats. Pour les rédacteurs de la tribune, il est très clair qu'on a dû «renoncer aux illusions qui avaient présidé à la création de la fonction cantonale. Le bon patriarche conciliateur qu'on avait rêvé doit être relégué au rang des utopies ».

13 P. TEMPLIER, De la tentative de conciliation, thèse droit, Paris 1898, conclusion, p. 311-317.

${ }^{14}$ Pour reprendre l'expression de l'historien américain Eugen Weber qui a consacré à l'administration de la justice dans les campagnes françaises un suggestif chapitre : «De la Justice, Seigneur, délivrez-nous ? », La fin des terroirs, Paris, Fayard, 1983, p. 85-107. 


\section{Jacques Poumarède}

Dans l'esprit de ceux qui en étaient chargés, la mort de la conciliation était annoncée. Elle fut prononcée à la sauvette par la loi du 9 février 1949 qui supprima la formalité du préliminaire de conciliation. Pierre Hébraud, éminent spécialiste de procédure civile à la faculté de droit de Toulouse, qui lui était plutôt favorable, prononça son Requiem en évoquant « le sacrifice de la conciliation judiciaire $»^{15}$. La "petite conciliation » instituée par les lois de 1838 et 1855 restait en place mais de moins en moins tentée et elle fut transférée au nouveau tribunal d'instance par la réforme judiciaire de 1958 qui supprima les juges de paix ${ }^{16}$.

La promulgation en 1975 du nouveau code de procédure civile a-t-il favorisé une réapparition de la conciliation devant les juridictions civiles ? A priori, on aurait pu le penser au vu de la section VIII (art.21) de ses Dispositions liminaires: «Il entre dans la mission du juge de concilier les parties ». Une formule péremptoire qui est déclinée plus loin aux articles 127 à 131 qui disposent que « les parties peuvent se concilier d'elles-mêmes ou à $l^{\prime}$ initiative du juge tout au long de l'instance... au lieu et au moment que le juge estime favorable ». La conciliation est constatée dans un procès-verbal, dont des extraits peuvent valoir titre exécutoire. Le nouveau code a même désigné un acteur et un moment privilégiés pour sa réalisation : art. 768 «Le juge de la mise en état peut constater la conciliation, même partielle, des parties ». Enfin, devant le tribunal d'instance, la "petite conciliation » des juges de paix a repris du service sous l'aspect d'une « demande aux fins de tentative préalable de conciliation" (art. 830 et s.), à la forme très simple mais laissée au bon vouloir des parties.

Manifestement, les efforts des rédacteurs du code n'ont pas suscité un bien grand engouement tant de la part des juges que du côté des justiciables et dans les premiers commentaires réalisés au tournant des années 19701980 on retrouve à des degrés divers l'écho des désillusions du XIXe siècle $^{17}$.

\footnotetext{
${ }^{15}$ JORF, 10 février 1949, p. 1499 ; P. HEBRAUD, «Commentaire de la loi du 9 février $1949 »$, Dalloz, 1949, Législation, p. 269.

${ }^{16}$ F. BANAT-BERGER, «La réforme de 1958, la suppression des justices de paix », J.-G. PETIT (dir.), Une justice de proximité : la justice de paix 1790-1858, ouv. cité, p. 225-247 ; d'après le Compte général de la justice civile (1967, p. 475) : 28067 conciliations pour 272465 affaires appelées (10,3\%).

${ }^{17}$ Y. DESDEVISES, Dalloz. 1981, Chronique, p. 241 « La conciliation dans le nouveau code »; G. BOLARD, «De la déception à l'espoir : la conciliation », Mélanges Pierre Hébraud, Toulouse, Univ. Sc. Sociales de Toulouse, 1981, p. 4657 ; P. ESTOUP, D. 1986, Chronique, «Etudes et pratique de la conciliation », p. 161. Nous n'évoquons pas ici la conciliation dans la procédure du divorce
} 
À la même époque, la création subreptice des « conciliateurs " est venue rajouter une poignée de sel dans le brouet judiciaire. L'idée de confier à des simples particuliers la mission de «faciliter, en dehors de toute procédure judiciaire, le règlement amiable des différends » est apparue en 1976 dans les cartons de la chancellerie à l'époque d'Olivier Guichard, ministre de la Justice du gouvernement de Raymond Barre. Elle s'inscrivait dans la préoccupation de faciliter l'accès à la justice et allait de pair avec la réforme de l'aide judiciaire. Le projet Guichard a pris corps, au début de 1977, par une simple note d'information de la chancellerie, publiée à la Revue des Huissiers, qui annonçait la nomination dans quatre départements d'une poignée de ces conciliateurs ${ }^{18}$ et, l'année suivante, sous son successeur, Alain Peyrefitte, un décret du 20 mars 1978 donnait en dix articles un cadre statutaire à cette institution ${ }^{19}$. Nommés pour un an renouvelable par le premier président de la cour d'appel, les conciliateurs ne devaient exercer aucune activité judiciaire à titre professionnel, ni être investis de mandat électif; leur fonction était bénévole et limitée à des différends de faible importance relevant normalement des tribunaux d'instance. Les rares commentaires de la doctrine, plutôt bienveillants, furent néanmoins dubitatifs sur la portée et le succès de l'institution: "Curieuse institution que le conciliateur!», écrit le professeur Jestaz... « en un mot comme en mille, le présent décret, d'ailleurs fort bref, n'accorde aucun pouvoir de droit au conciliateur et se borne à lui reconnaître les prérogatives de pur fait dont tout un chacun dispose déjà de par la déclaration des droits - laquelle permet ce que la loi n'interdit pas $»^{20}$.

Du côté de la magistrature l'accueil fut plus frais, voire franchement hostile. Il faut rappeler que les rapports entre le garde des Sceaux, Alain Peyrefitte et le corps de la magistrature traversaient à l'époque une phase de tension et la création des conciliateurs a été perçue comme une sorte de désaveu à l'égard du labeur quotidien des juges les plus modestes de la hiérarchie judiciaire: les juges d'instance, et un moyen de combler à moindre frais la pénurie des effectifs. Le plus en pointe dans la contestation, le Syndicat de la magistrature, a traité la question par la dérision : «Sous

(NCPC, art. 1093-1108) ou celles prévues devant certaines juridictions spéciales, comme les conseils des prud'hommes ou les tribunaux paritaires des baux ruraux.

${ }^{18}$ Revue des Huissiers, 1977, IV, p. 43, une manière pour le moins discrète et inhabituelle d'annoncer les choses. Les départements en cause: les AlpesMaritimes, la Gironde, la Haute-Marne et la Loire-Atlantique.

19 JORF, 23 mars 1978, p. 1265 : décret $n^{\circ} 78-381$ du 20 mars 1978 relatif aux conciliateurs.

${ }^{20}$ Revue trimestrielle de droit civil, 1978, p. 755. 


\section{Jacques Poumarède}

prétexte de dégager les juges d'instance de leur surcroît de travail, l'instauration généralisée des conciliateurs constitue un retour évident du système des notables de 1790, sans toutefois le contrôle du suffrage universel, puisque ces «magistrats» sont désignés par les premiers présidents des cours d'appel $»^{21}$. Quant à l'organisation majoritaire, l'Union syndicale de la magistrature qui avait pris la suite en 1974 de l'U.F.M., son opinion sur le projet Guichard, bien que plus argumentée, n'en était pas moins sévère comme en témoigne la conclusion d'un rapport présenté au congrès de 1978 : «En définitive, ce projet est un gadget et, dans certaines circonstances, il peut se révéler un jouet dangereux $»^{22}$.

Devant cette fronde, le ministère de la Justice fit réaliser en 1981 par le Centre de recherche pour l'étude et l'observation des conditions de vie (CREDOC) un rapport sur " Le rôle du conciliateur et ses relations avec la justice ", sur la base d'une enquête par entretiens auprès d'un échantillon de conciliateurs, de magistrats, d'auxiliaires de justice, d'élus et de responsables associatifs. Au vu des résultats, on y perçoit des opinions assez divergentes à l'égard d'une institution encore très jeune. Si les chefs de cours, premiers présidents et procureurs généraux, se déclarent satisfaits, responsabilités hiérarchiques obligent, les autres magistrats se montrent plus hésitants et certains franchement hostiles: " Il n'y a pas besoin de conciliation dans la population (...). Dans le contexte actuel, la conciliation, c'est du luxe !! ». Le rapport souligne: « une attitude a priori réservée des juges d'instance» qui «ne pensent pas que, dans tous les cas, une conciliation soit préférable à un jugement » et qui la perçoivent comme une forme de «dépossession» d'une partie de leur fonction. À travers certaines positions, on retrouve l'écho des préjugés contre les juges de paix du XIXe siècle et les mêmes doutes sur leurs aptitudes à remplir la mission qu'on leur avait confiée : «Concilier est une opération difficile qui exige de la part de celui qui entend la mener à bien beaucoup d'expérience ainsi qu'une formation juridique sérieuse ». Seule une minorité des juges interrogés se montre assez favorable à cette institution, le plus souvent par "réalisme ». Quant au barreau, les positions sont globalement négatives, oscillant, d'après le rapport, "entre un scepticisme sur l'avenir de cette nouvelle

\footnotetext{
21 Justice (bulletin du S.M.), $\mathrm{n}^{\circ}$ 84, mars-avril 1982, Didier MARCHALL, «Le tribunal d'instance : un enjeu », p. 10.

${ }^{22}$ Le Nouveau Pouvoir judiciaire, ${ }^{\circ}$ 280, p. 19-23, rapport de M. Eugène Clavel, conseiller à Rouen au nom de la commission sur l'accès à la justice.
} 
institution, et une hostilité qui s'appuie sur les inconvénients qu'elle peut représenter pour les justiciables $»^{23}$.

$\mathrm{Au}$ vu de ces réactions, on ne s'étonne pas d'apprendre que l'expérience des conciliateurs fut à deux doigts d'être arrêtée à l'époque où Robert Badinter occupait les fonctions de ministre de la Justice dans le cabinet Mauroy $^{24}$. Elle sera néanmoins maintenue contre l'assurance que leur activité purement bénévole serait cantonnée en dehors de toute procédure judiciaire. Un temps suspendu, le recrutement des conciliateurs a repris sous le garde des Sceaux Albin Chalandon (1987-1988).

C'est au cours des années 1990 que la conciliation a connu un regain d'intérêt et une évolution de sa pratique qui ont amorcé un processus dont nous présentons, en guise de conclusion les principales étapes.

Un premier signe de changement a été apporté par un décret du 25 février 1993 qui a exigé des futurs conciliateurs « une expérience d'au moins cinq ans en matière juridique $»^{25}$. Mais c'est surtout la loi du 8 février 1995 sur l'organisation judiciaire, et ses décrets d'application qui ont changé la donne. Pour faire face à une inflation considérable des taches assumées par les juges, le législateur a pensé trouver la solution en les autorisant à se faire assister par des tierces personnes spécialisées et même à déléguer une partie de leur compétence, notamment en matière de conciliation. Les conciliateurs étant déjà en place, le principe posé par l'article 21 de la loi du 8 février 1995 a été décliné par le décret du 22 juillet 1996 qui a donc autorisé le juge d'instance à désigner éventuellement l'un d'entre eux pour le suppléer dans la tentative préalable de conciliation toujours en usage devant le tribunal $\mathrm{d}^{\prime}$ instance ${ }^{26}$.

${ }^{23}$ CREDOC, Le rôle du conciliateur et ses relations avec la justice, 1981, rapport dactylographié, 115 p., voir les extraits d'entretiens rassemblés aux p. 87 à 102.

${ }^{24}$ Selon le témoignage apporté au cours du présent colloque par M. le premier président Jean-Pierre Pech, qui était à l'époque conseiller au cabinet du garde des Sceaux.

${ }^{25}$ JORF, $\mathrm{n}^{\circ} 49,27$ fév. 1993, D. $\mathrm{n}^{\circ}$ 93-254, art. 1-2.

${ }^{26} J O R F, \mathrm{n}^{\circ} 170,23$ juillet 1996, p. 11125, D. n 96-652, «art. 831 : La tentative préalable de conciliation peut être menée par le juge ou par un conciliateur remplissant les conditions prévues par le décret du 20 mars 1978 relatif aux conciliateurs désignés à cet effet». En conséquence, le même décret a modifié l'article 830 et suivants du code de procédure civile; M. OLIVIER, « La 


\section{Jacques Poumarède}

Les conciliateurs pouvaient ainsi sortir des salles municipales où ils exerçaient habituellement leurs modestes activités et franchir les portes des temples de Thémis, en qualité d'auxiliaires; d'ailleurs quelques mois plus tard, un nouveau décret leur donnait le titre de "conciliateur de justice » tout en accordant habilement aux juges d'instance un droit de proposition sur leur nomination et leur reconduction ${ }^{27}$. Cette évolution statutaire n'a pas suscité d'hostilité particulière de la part des juges professionnels, qui auront bientôt d'autres occasions de se mobiliser, notamment contre l'introduction de la «justice de proximité ». Les conciliateurs de justice ont continué leur bonhomme de chemin, en se consacrant surtout à la conciliation extrajudiciaire car les délégations ont très vite plafonné à moins de $10 \%$ de leur activité totale.

La réforme des années 1995-1996 a tout de même introduit une autre pomme de discorde avec la reconnaissance de la pratique de la "médiation judiciaire ». Toujours dans le but d'alléger leurs charges, la loi du 8 février 1995 et le décret du 22 juillet 1996 ont autorisé les juges à désigner des tierces personnes ou des associations spécialisées à intervenir dans des affaires particulièrement conflictuelles pour tenter de rapprocher les parties à l'aide des techniques de la communication; les affaires matrimoniales ou familiales étaient particulièrement visées mais la loi n'a pas donné de cadre bien délimité à cette procédure et la médiation est devenue assez vite un terrain d'activités professionnalisées et plus ou moins lucratives ${ }^{28}$.

Les derniers développements de cette saga viennent de se produire. Une conjonction de facteurs a suscité un mouvement en faveur du changement. Il y a d'abord le malaise, pour ne pas parler de crise, dans lequel se trouvent les institutions judiciaires. Le moment n'est plus à la désunion mais à la recherche de solutions. Parmi les expertises réalisées, l'une a retenu particulièrement l'attention : il s'agit du rapport rédigé, à la demande de Rachida Dati, garde des Sceaux, par une commission placée sous la présidence du recteur Guinchard. Remis en 2008 sous un titre très explicite : L'ambition raisonnée d'une justice apaisée, il présente 65 propositions de

conciliation et la médiation judiciaires en matière civile (aspects anciens et actuels) », Gazette du Palais, 19 oct. 1996, p. 1257-1261.

${ }_{27}$ JORF, $\mathrm{n}^{\circ} 292,15$ déc. 1996, p. 18424, D. $\mathrm{n}^{\circ}$ 96-1091, art. $1^{\mathrm{er}}$; l'article réduit à 3 ans la durée de l'expérience juridique.

${ }^{28}$ La médiation a été, elle aussi, introduite dans le code de procédure civile aux articles 131-1 à 131-10; Juris-classeur Procédure civile, «Conciliation et médiation », par P. COUVRAT et G. GIUDICELLI-DELAGE ; sur cette pratique, voir la synthèse de M. GUILLAUME-HOFNUNG, La médiation, Paris, PUF, Que

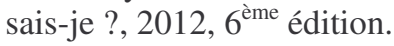


réforme, parmi lesquelles figurent plusieurs mesures pour favoriser le développement des modes alternatifs de règlement des conflits ${ }^{29}$. L'idée défendue est d'ouvrir plus largement la porte du procès civil aux conciliateurs comme aux médiateurs mais sous la responsabilité et le contrôle des magistrats professionnels : «un juge décideur, au cœur d'une équipe ».

Dans le même temps mais à l'initiative du premier président de la cour d'appel de Paris, Jean-Claude Magendie, et du directeur de l'Ecole nationale de la magistrature, Jean-François Thony, un groupe de travail s'est penché spécialement sur les conciliateurs de justice et à abouti à des préconisations qui rejoignent et précisent celles du rapport Guinchard ${ }^{30}$. L'implication de ces hautes personnalités de la magistrature a été appréciée comme il se devait par les principaux intéressés. Les effets de ces prises de position se sont concrétisés dans un décret du $1^{\text {er }}$ octobre 2010 relatif à la conciliation et à la procédure orale ${ }^{31}$. Le champ d'intervention des conciliateurs par délégation du juge a été étendu : ils sortent du préliminaire de conciliation de la procédure d'instance où ils étaient cantonnés pour intervenir tout au long de la procédure et théoriquement à tous les niveaux des juridictions civiles, sous le contrôle du juge dont ils deviennent de véritables collaborateurs. En contrepartie, la forme de l'acte de conciliation a été mieux précisée et l'encadrement de leur recrutement et de leurs activités par les autorités des cours d'appel a été renforcé. Le même décret leur a également ouvert les portes des tribunaux de commerce et des tribunaux paritaires des baux ruraux ${ }^{32}$.

Enfin, n'en déplaise aux mânes de Thouret et de Treilhard, le droit français est aujourd'hui tributaire des normes définies par les instances

${ }^{29}$ S. GUINCHARD, L'ambition raisonnée d'une justice apaisée : rapport au garde des Sceaux, Paris, Documentation française, 2008, voir notamment p. 128-132 : "section 1 - Le développement et la consolidation de l'institution des conciliateurs de justice ».

30 J.-Cl. MAGENDIE et J.-F. THONY, Célérité et qualité de la justice: les conciliateurs de la justice, Paris, La Documentation française, 2010, 130 p. Voir les préconisations p. 112-113: le rapport conseille une meilleure «implication des magistrats chargés de suivre l'activité des conciliateurs », des cycles de formations proposés par l'ENM, le maintien d'une « grande souplesse dans la facilitation de la solution » pour les conciliateurs, mais le rappel «des devoirs » qu'imposent leurs fonctions, etc.

${ }^{31}$ JORF, $\mathrm{n}^{\circ}$ 0230, 3 octobre 2010, p. 17986 ; Gazette du Palais, 10-12 oct. 2010, N. GERBAY, « Premières vues sur le décret du $1^{\text {er }}$ octobre $2010 »$, p. 17-20.

${ }^{32}$ Le même décret a intégré ces dispositions dans le code de procédure civile aux articles 129-1 à 5, 130, 131, 312, 862-2 et 887-2. 


\section{Jacques Poumarède}

européennes. Or, depuis 1999, au nom du principe fondamental de libre accès à la justice, le Conseil européen a invité les États membres à développer les modes alternatifs de résolution des conflits et notamment la médiation en la présentant comme "une solution extrajudiciaire économique et rapide aux litiges en matière civile et commerciale ». Du «Livre vert » présenté sur le sujet par la Commission (2002) en passant par les avis et les débats tenus devant le Parlement européen (2007), le processus a débouché sur une directive adoptée le 21 mai $2008^{33}$. Le principe de l'accès à la justice ne masque pas la promotion d'intérêts bien évidents. La médiation est devenue une activité créatrice de services et d'emplois, producteurs eux-mêmes de systèmes de formation et de sources de revenus. Les lobbies concernés n'ont pas manqué de pousser à la roue communautaire.

La France a pris son temps pour transposer cette directive, car si la médiation a déjà sa place dans notre droit depuis 1995, l'existence des conciliateurs de justice est une exception française qui pouvait paraître à la marge du cadre de la directive 2008/52/CE. Il n'empêche, une ordonnance de transposition a été prise le 16 novembre $2011^{34}$ et dans la foulée un décret d'application a été signé le 20 janvier $2012^{35}$. Ce texte opère une novation importante en créant dans le code de procédure civile in fine un livre $\mathrm{V}$ consacré à «La résolution amiable des différends ». Sous cette dénomination sont regroupées la conciliation extrajudiciaire pratiquée par les conciliateurs de justice, la médiation conventionnelle et, pour faire bonne mesure, la "procédure participative ", c'est-à-dire une transaction entre parties réalisée par l'intermédiaire de leurs avocats. Le décret ne cherche pas à établir de distinction et donne aux deux premières pratiques la même définition: (CPC, art. 1530) « La médiation et la conciliation conventionnelles régies par le présent titre s'entendent de tout processus structuré, par lequel deux parties tentent de parvenir à un accord, en dehors de toute procédure judiciaire en vue de la résolution amiable de leurs différends, avec l'aide

\footnotetext{
${ }^{33}$ Journal officiel de l'Union européenne, 24 avril 2008, L 136 1-8, sur le site eurlex.europa.eu

${ }^{34}$ JORF, 17 nov. 2011, p. 19286 et s., Ordonnance de transposition et rapport au président de la République; B. GORCHS-GELZER, «Regard critique sur l'ordonnance $\mathrm{n}^{\circ}$ 2011-1540 transposant la directive médiation», Droit et Procédures, 2012, $\mathrm{n}^{\circ}$ 1, Chronique, p. 1 à 5 . De la même auteure, voir une réflexion d'ensemble sur «La conciliation comme 'enjeu' dans la transformation du système judiciaire », Droit et société, 2006, n 62, p. 223-256.

${ }_{35}$ JORF, 22 janv. 2012, p. 1280, décret $\mathrm{n}^{\circ}$ 2012-66 relatif au règlement amiable des différends.
} 
d'un tiers choisi par elles qui accomplit sa mission avec impartialité, compétence et diligence ».

Désormais la conciliation est pleinement reconnue dans tous ses aspects : qu'elle soit judiciaire ou conventionnelle, elle est gravée sur les tables du code. Que lui reste-il pour se faire enfin aimer des juges?

Depuis 2003, il existe un réseau dénommé GEMME (Groupement européen de magistrats pour la médiation) dont le président fondateur fut Guy Canivet, premier président de la Cour de cassation, et qui a beaucoup œuvré pour la transposition en droit français de la directive européenne ${ }^{36}$. Le site Internet de cette association ( $\underline{w w w}$.gemme.eu $)$ comprend depuis peu dans le fonds documentaire de la partie FRANCE une nouvelle rubrique intitulée «conciliation» qui donne accès au guide méthodologique des conciliateurs de justice publié récemment par la direction des services judiciaires du ministère de la Justice. Signe des temps : la conciliation sera-telle sauvée de la « désunion » par les $\mathrm{TIC}^{37}$ ?

\footnotetext{
${ }^{36}$ On peut lire les positions du GEMME sur le décret du 20 janvier 2012 dans Les Annonces de la Seine, supplément au n ${ }^{\circ} 27$ du jeudi 19 avril 2012.

${ }^{37}$ Les techniques de l'information et de la communication : information fournie par M. Gilbert Cousteaux, président de chambre à la cour d'appel de Toulouse, membre du GEMME et participant à la gestion du site, que nous remercions.
} 